\title{
FLORA LIQUÉNICA SAXÍCOLA Y ARENÍCOLA DE LA PENÍNSULA DE O GROVE (PONTEVEDRA, NW DE ESPAÑA)
}

\author{
Montserrat FREIRE DOPAZO y Aida GARCÍA MOLARES
}

\begin{abstract}
RESUMEN. Flora liquénica saxícola y arenícola de la Península de O Grove (Pontevedra, $N W$ de España). En el presente trabajo se expone el catálogo de la flora liquénica saxícola y arenícola de la Península de $\mathrm{O}$ Grove, promontorio rocoso localizado en la costa de la provincia de Pontevedra y unido al continente por un istmo arenoso de reciente formación. Entre los 188 taxa identificados, Thelotrema wightii (Taylor) Nyl. y Caloplaca ceracea Laundon son novedad para la flora española, otros 19 taxa suponen un incremento del catálogo de líquenes de la comunidad gallega y 37 más constituyen novedad para la provincia de Pontevedra. Además se amplía la corología de algunas especies raramente citadas en la Península Ibérica. En este trabajo también se lleva a cabo un análisis de la zonación de la vegetación liquénica apreciada en las localidades litorales del área de estudio.
\end{abstract}

Palabras clave. Flora liquénica, saxícola, arenícola, zonación litoral, O Grove, Galicia.

RÉSUMÉ. Flore lichénique saxicole et arénicole de la Péninsule de O Grove (Pontevedra, NW España). Dans ce travail on presente le catalogue de la flore lichénique saxicole et arénicole de la péninsule de $\mathrm{O}$ Grove, promontoire rocheux dans la côte de Pontevedra, rattaché au continent par un isthme sablonneux d'origine recent. Parmi les 188 taxa identifiées, on découvre la première citation pour la flore espagnole sur Thelotrema wightii (Taylor) Nyl. et Caloplaca ceracea Laundon, 19 taxa qui grossisent le catalogue de lichens de la Galice et d'autres 37 inconnus dans la province de Pontevedra. En outre, on augmente la chorologie de quelques espèces peu souvent citées dans la Péninsule Ibérique. Dans ce travail on analyse aussi la zonation de la végétation lichénique enregistrée dans le littoral de l'aire d'étude.

Mots clés. Flore lichénique, saxicole, arénicole, zonation littoral, O Grove, Galice.

\section{INTRODUCCIÓN}

El estudio de la flora liquénica en Galicia se aborda de forma continuada a partir de la década de los ochenta (Carballal et al., 1983, 1985; Carballal \& López de Silanes, 1985; Carballal \& García Molares, 1986, 1987, 1987 88, 1988; Bahillo et al., 1987; Álvarez \& Carballal, 1987, 1988; etc.); hasta ese momento sólo existían referencias fragmentarias (Lange,
1860; Amo \& Mora, 1870; Colmeiro, 1889). Sin embargo, y a pesar de las numerosas aportaciones realizadas desde entonces, puede afirmarse que el catálogo de líquenes dista mucho de ser completado. Todavía permanecen inexplorados muchos territorios de nuestra región y no se conoce bien la corología de las especies.

Aunque los catálogos más extensos corresponden al estudio de líquenes epifíticos 
(Carballal et al., 1983, 1985; Carballal \& López de Silanes,1985; Carballal \& García Molares, 1986, 1987, 1987-88, 1988; Álvarez \& Carballal, 1987, 1988; etc.), en los últimos tiempos se ha intensificado el estudio de la flora saxícola (Pérez et al., 1991; Etayo et al., 1991; López de Silanes, 1992; Valcárcel et al. 1992; Valcárcel, 1994; Prieto et al., 1994, 1995). En este contexto, los trabajos en tramos litorales son aún escasos. Sampaio \& Crespí (1927) elaboran un amplio catálogo de líquenes de la provincia de Pontevedra que incluye numerosas localidades costeras y en algunas publicaciones antiguas aparecen citas puntuales de la costa gallega (Lange, 1860; Follman, 1973; Fischer-Piette \& Seoane-Camba, 1962; Pérez-Cirera, 1976), si bien los trabajos de Bahillo et al. (1987), Sánchez-Biezma (1997) y Paz-Bermúdez (1998) son los únicos que se refieren exclusivamente a la flora liquénica del litoral gallego. Las prospecciones realizadas en estos ambientes han mostrado una riqueza florística inusitada y la presencia de un buen número de especies interesantes, debido a la diversidad de hábitats que presentan.

\section{MATERIAL Y MÉTODOS}

La Península de O Grove se encuentra situada en la provincia de Pontevedra, al sur de la ría de Arousa; está unida al brazo de tierra que separa las rías de Pontevedra y Arousa a través de un tómbolo arenoso, A Lanzada, cuyo origen se remonta a un período histórico reciente (en las cartas marítimas del siglo XVI se menciona todavía la "isla de El Grove").

Presenta una orografía accidentada, si bien no se alcanzan cotas muy elevadas. Los montes más altos se encuentran en la parte oriental, en la parroquia de S. Martiño (Monte Loureiro, $167 \mathrm{~m}$; A Siradella, $159 \mathrm{~m}$ ). Separada por una zona central baja, llana y alterada, que pone en evidencia la existencia de una erosión diferencial, la parte occidental de la isla en ningún caso sobrepasa los $100 \mathrm{~m}$ de altitud.

La franja costera es muy irregular, alternando salientes rocosos, con pendientes relativamente fuertes, y zonas de playa más o menos amplias.

El territorio es muy homogéneo desde el punto de vista geológico. Se trata mayoritariamente de rocas ígneas ácidas (granodioritas) aunque también aparecen rocas metamórficas (esquistos y gneises) correspondientes al complejo Cabo Home-A Lanzada. Es importante destacar la presencia de depósitos de distinto tipo, tales como dunas, arenas de playa, depósitos colubiales y un cono de deyección distribuidos a lo largo de la costa ocupando las zonas llanas.

En cuanto a los suelos son, según la clasificación FAO, tipo Cambisol o Leptosol. También aparecen Arenosoles a lo largo de la costa y suelos hipersalinos limosos (incluidos en distintos grupos en la clasificación FAO) en la zona del istmo.

Es preciso señalar que gran parte del territorio, al igual que el resto de las áreas costeras gallegas, se encuentra sometida a una importante influencia antropogénica (modificación de la costa, nitrificación).

El muestreo se limitó a los substratos rocosos y arenosos; en algunas localidades también se tomaron muestras sobre suelo poco compactado o sobre mortero. Fueron seleccionadas 10 localidades de las cuales 6 son estrictamente costeras y 4 interiores, más alejadas de la influencia marina (fig. 1). En las localidades litorales se ha tenido en cuenta la geomorfología costera, los niveles alcanzados por las mareas y el grado de exposición al oleaje; en todos los transectos efectuados, se ha estudiado por separado los tramos correspondientes al mesolitoral, supralitoral y adlitoral (Seoane-Camba, 1969), con el fin de delimitar su flora liquénica. El mesolitoral o zona intermareal está situado entre el nivel más alto y más bajo alcanzado por las mareas; el supralitoral está situado por encima del nivel 


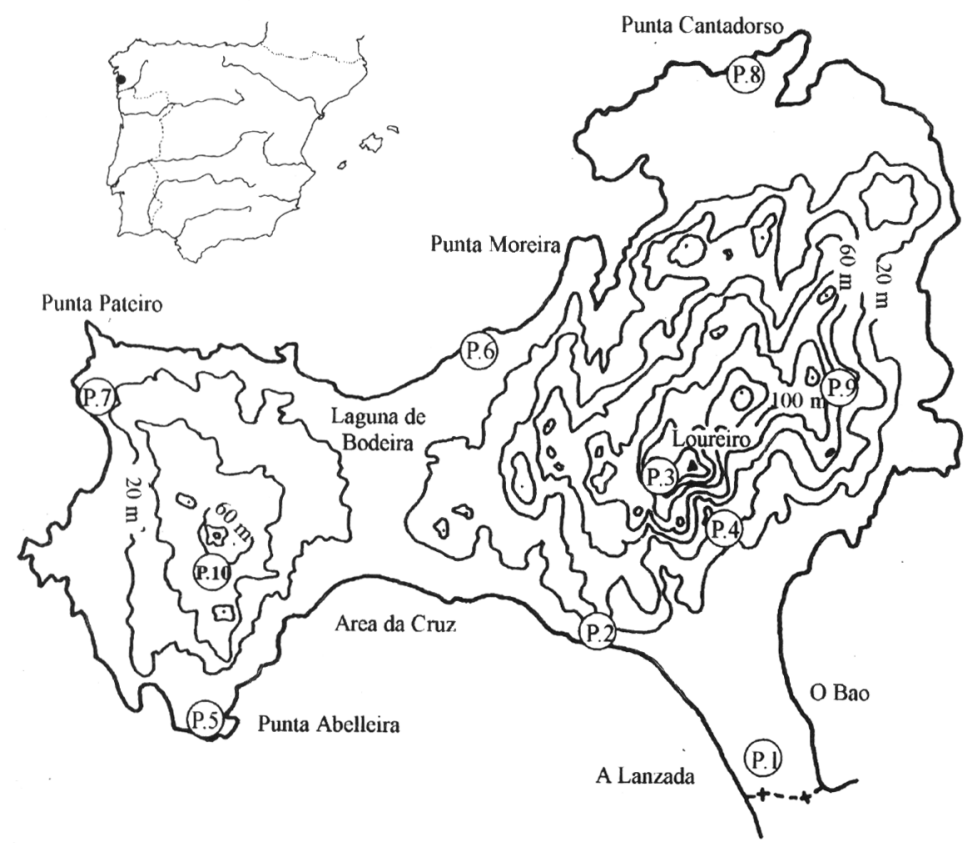

Figura 1. La península de O Grove: situación de las localidades prospectadas.

más alto alcanzado por las mareas y la zona subterrestre o adlitoral se extiende desde el límite de instalación de la vegetación superior, que progresivamente se hace dominante a medida que nos alejamos de la costa.

La relación de localidades prospectadas es la siguiente:

1.- Playa de A Lanzada, 29TNG1099, dunas bajas, substrato arenoso más o menos consolidado.

2.- Proximidad punta de Raeiros, 29TNH0900, substrato rocoso (granodiorita).

3.- Monte de A Siradella, 29TNH0902, $159 \mathrm{~m}$ de altitud, afloramientos rocosos (granodiorita) y suelo (leptosol).

4.- Cantera cerca de Molino, 29TNH0902, 30 $\mathrm{m}$ de altitud, substrato rocoso (granodiorita).

5.- Punta Abelleira, 29TNH0600, afloramientos rocosos (granodiorita) y suelo (Leptosol) en supralitoral y adlitoral; esquistos o gneises en el mesolitoral.
6.- Playa de A Mexilloeira, 29TNH0803, afloramientos rocosos (granodiorita en adlitoral y supralitoral; esquistos o gneises en el mesolitoral); mortero en construcción próxima. 7.- Punta Pateiro, 29TNH0502, substrato rocoso (granodiorita en adlitoral y supralitoral; gneises en mesolitoral).

8.- Punta Cantadorso, 29TNH1005, substrato rocoso (granodiorita); mortero en construcción próxima.

9.- Ardía, cerca vertedero de basuras, 29TNH1103, $60 \mathrm{~m}$ de altitud, substrato rocoso (granodiorita).

10.- Proximidad recinto militar de Jarda, 29TNH0601, substrato rocoso (grandes bloques graníticos).

La toma de muestras, conservación e identificación del material se llevó a cabo de acuerdo con los procedimientos habituales en Liquenología. En lo relativo a la nomenclatura 
taxonómica se ha seguido a Purvis et al. (1992), recurriendo a Clauzade \& Roux (1985, 1987, 1989) y Nimis (1993) para aquellas especies que no aparecen en la primera de las obras señalada. Para la identificación de algunos géneros conflictivos, como Rinodina y Rhizocarpon, entre otros, se han tenido en cuenta los trabajos monográficos más recientes (Giralt \& Barbero, 1995; Giralt et al., 1997; Mayrhofer et al., 1993; Poelt, 1988, etc.), adoptándose los cambios nomenclaturales propuestos. El material se encuentra depositado en el herbario VIGO-Lich. de la Universidad de Vigo.

\section{RESULTADOS}

A lo largo del estudio se ha confeccionado un catálogo de 188 taxa liquénicos. Las especies identificadas y las localidades donde han sido recogidas se muestran en la tabla 1 . Por su interés corológico, cabe destacar la presencia de Thelotrema wightii (Taylor) Nyl. y Caloplaca ceracea Laundon, nuevos para la flora española, y con una distribución muy restringida en el ámbito europeo. Hasta la fecha sólo existían referencias antiguas de la existencia de pequeñas poblaciones de $T$. wightii en cuatro localidades del norte de Portugal (Nylander, 1888; Tavares, 1956; Purvis et al., 1995). Caloplaca ceracea se conocía, únicamente, de localidades costeras del oeste de las Islas Británicas (Laundon, 1992). El catálogo incluye además 18 especies y 1 subespecie no detectadas en Galicia y 37 nuevas citas más para la provincia de Pontevedra. Asimismo, se amplía la distribución de algunos taxa raramente identificados en la Península Ibérica; tal es el caso de Lecania aipospila y Pertusaria lactescens que se conocen de la provincia de A Coruña (Paz - Bermúdez, 1998 y Sánchez Biezma, 1997), Cladonia metacorallifera conocida de Lugo (Valcárcel et al., 1991;
Álvarez, 1993; Valcárcel, 1994) y Lecania hutchinsiae citada de A Coruña (Sánchez Biezma, 1997; Paz - Bermúdez, 1998), Lugo y Pontevedra (Paz - Bermúdez, 1998). De Acrocordia macrospora, Caloplaca crenulatella y Leproloma cacuminum se conocen citas de localidades gallegas (Paz Bermúdez, 1998; Sánchez - Biezma, 1997) y de Cataluña (Llimona, 1991; Navarro Rosinés, 1992, Boqueras, 1993); Lecania atrynoides y Toninia mesoidea se conocen de localidades de la comunidad gallega (Paz Bermúdez, 1998; Sánchez - Biezma, 1997) y de Asturias (Boom \& Gómez - Bolea, 1991; Timdal, 1991; Paz - Bermúdez, 1998); Cladonia rei está citada de Navarra y Cataluña (Burgaz \& Ahti, 1994). Finalmente, Opegrapha caesareensis, Roccella tuberculata, Lecidella scabra, Ochrolechia crozalsiana, Leptogium massiliense y Rhizocarpon drepanodes se mencionan muy raramente en la bibliografía.

La mayoría de los taxa identificados se encuentran ampliamente distribuidos en el continente europeo como por ejemplo Xanthoria parietina, Anaptychia runcinata o Candelariella vitellina. El catálogo recoge un buen número de especies oceánicas, de las cuales Nephroma tangeriense, Opegrapha calcarea, Pertusaria monogona, Trapeliopsis pseudogranulosa y Parmelia revoluta var. britannica se extienden por diferentes áreas de la porción occidental de Europa (Purvis et al., 1992; Nimis, 1993; etc.).

Muchas de las especies características de las localidades litorales de la Península de $\mathrm{O}$ Grove muestreadas como Caloplaca littorea, C. marina, C. thallincola, Lecanora gangaleoides, Pertusaria gallica, P. rupestris, Roccella fuciformis, Verrucaria maura, etc. se distribuyen a lo largo de las costas atlántica y mediterránea. Caloplaca microthallina, Cladonia squamosa var. subsquamosa, Lecania aipospila, Lecanora actophila, Opegrapha caesareensis, Ramalina siliquosa, Rinodina beccariana var. beccariana y Verrucaria 
prominula están confinadas, sin embargo, al litoral atlántico europeo. Frente a un amplio número de taxa liquénicos distribuidos hacia el centro y regiones meridionales de Europa tales como Buellia stellulata, Diploschistes actinostomus, Lecidella viridans, Parmelia revoluta, Parmotrema reticulatum, Pertusaria pseudocorallina, Rhizocarpon richardii, Scoliciosporum umbrinum, etc., cabe destacar la presencia de Aspicilia calcarea, Buellia sardiniensis, Cladonia convoluta, Collema ryssoleum, Diploschistes diacapsis subsp. interpediens, Lecanora poeltiana, L. schistina, Parmelia tinctina, Petractis thelotremella y Protoparmelia montagnei, con un marcado carácter mediterráneo. Finalmente, un pequeño grupo de especies presenta una distribución más restringida en el ámbito continental, además de los ya mencionados Thelotrema wightii y Caloplaca ceracea. Roccella boergesenii se encuentra en la región macaronésica y costa portuguesa. Leptogium microphylloides ha sido citado con anterioridad en distintos puntos de la geografía francesa y todas las provincias gallegas, al igual que Ochrolechia crozalsiana, conocida solamente en Francia y alguna localidad española. Rhizocarpon drepanodes, Lecanora lecideoides y Pertusaria lactescens se presentan también de forma puntual y su distribución es mal conocida.

La mayoría de las especies identificadas fueron recogidas sobre granodiorita, dado que es el substrato predominante en la zona de estudio, si bien Opegrapha caesareensis y Porina chlorotica se presentan directamente sobre granos gruesos de turmalina, mineral rico en bases asociado a este tipo de roca. Sobre los escasos afloramientos de esquistos y gneises situados en el mesolitoral de algunos puntos de muestreo hemos localizado Lecania nylanderiana, Lecanora poeltiana, Porpidia macrocarpa, Protoparmelia montagnei, Pyrenocollema halodytes y Verrucaria prominula, además de otros líquenes propios de ambientes costeros que se desarrollan sobre cualquier tipo de roca; tal es el caso de Caloplaca marina, C. thallincola, Lecania aipospila, L. atrynoides, Lecanora helicopis, Lichina confinis y Verrucaria maura, entre otros.

Hemos identificado cierto número de especies propias de substratos calcáreos, según datos bibliográficos, sobre rocas ácidas cuya presencia se justifica si consideramos el enriquecimiento en sales que presenta la superficie rocosa propiciado por su proximidad al mar. Entre ellas cabe destacar Acrocordia salweyi, Aspicilia calcarea, A. contorta, Catillaria lenticularis, Collema cristatum, $C$. ryssoleum, Leptogium tenuissimum, Petractis thelotremella y Toninia mesoidea.

Algunas especies como Caloplaca aurantia, Collema undulatum, Thelidium minutulum y Verrucaria nigrescens se recogieron exclusivamente sobre mortero, substrato especialmente rico en sales de calcio, lo que denota el marcado carácter calcícola de estos taxa frente al resto de la flora catalogada. Un buen número de especies como Cetraria aculeata, Degelia atlantica, Hypogymnia physodes, Leptogium teretiusculum, Parmelia revoluta y la mayor parte de las especies de Cladonia se presentan en suelo póximo a la base de las rocas muestreadas o rellenando grietas o impregnando las mismas. Cladonia rangiformis var. rangiformis y var. pungens se localizaron indistintamente en suelo tipo Leptosol o arena y tan sólo tres especies (Cladonia convoluta, C. subrangiformis y Thermutis velutina) aparecen exclusivamente en substrato arenoso.

Algunas de las especies que incluye el catálogo se encuentran formando parte de comunidades brioliquénicas, en unos casos de forma ocasional y en otros de manera constante. En este último grupo se sitúan Cladonia caespiticia, C. symphycarpa, Collema auriforme, Leptogium gelatinosum, las dos variedades identificadas de $L$. lichenoides, $L$. 
massiliense, Parmelia horrescens y Leprocaulon microscopicum. El hecho de que estas especies se presenten asociados a briófitos indica un mayor grado de hidrofilia con respecto a las restantes, en este tipo de ambientes expuestos a una intensa insolación.

El estudio de la zonación en localidades litorales, ha arrojado los siguientes resultados: En el adlitoral, cabe destacar la presencia de Xanthoria parietina, Buellia stellullata, Candelariella vitellina, Lassalia pustullata, Lecanora gangaleoides, Ochrolechia parella, Parmotrema reticulatum, P. stuppeum, Ramalina siliquosa, Rhizocarpon richardii, Parmelia conspersa, P. caperata y Caloplaca crenularia. De forma más puntual, también fueron localizados Buellia saridiniensis, B. subdisciformis, Lecanora sulphurea, Lecidella scabra, Parmelia verruculifera, P. glabratula subsp. fuliginosa, P. pulla, y Parmotrema chinense. Roccella fuciformis y $R$. phycopsis, junto a otras especies del género menos abundantes, fueron recogidas en paredes verticales de localidades expuestas. En el supralitoral se encuentran bien representados Buellia stellulata, Anaptychia runcinata, Caloplaca crenularia, C. littorea, Candelariella vitellina, Catillaria chalybeia, Lecanora campestris, L. dispersa, Ochrolechia parella, Pertusaria pseudocorallina, Ramalina siliquosa, Tephromela atra y Xanthoria parietina. Menos abundantes, también aparecen Rhizocarpon richardii, Sarcogyne clavus, Scoliciosporum umbrinum, Diploschistes actinostomus, Lecanora actophila, Pertusaria gallica, Porpidia cinereoatra y Rinodina beccariana var. beccariana, entre otros. En este nivel también es importante la presencia de Caloplaca marina, próxima al límite superior de la marea. La zona sometida a la acción de las mareas, mesolitoral o litoral mésico, se caracteriza por la abundante presencia de Verrucaria maura, Caloplaca marina, C. thallincola y Lecania aipospila. Asociados a este nivel también aparecen Verrucaria prominula, Diplotomma ambiguum, Catillaria lenticularis, $C$. atomarioides, Pyrenocollema halodytes y, de confirmarse, Caloplaca microthallina. Lichina confinis y L. pygmaea son, también, características de este nivel, aunque han sido localizadas en zonas más expuestas; se presentan siempre muy próximas al límite inferior de la zona intermareal y, aunque pueden encontrarse mezcladas, L. confinis suele ocupar posiciones más elevadas que L. pygmaea.

\section{CONCLUSIONES}

La península de $\mathrm{O}$ Grove ha resultado un enclave extraordinariamente interesante desde el punto de vista liquenológico. Tras el estudio preliminar de los líquenes saxícolas y arenícolas se han catalogado un total de 188 taxa, cifra importante si se considera la limitación del ámbito geográfico y el número de localidades muestreado.

Entre las especies identificadas se incluyen Thelotrema wightii y Caloplaca ceracea, nuevas para la flora española y de distribución muy restringida en Europa. 18 especies y 1 subespecie constituyen novedad para la flora gallega. 35 especies, 1 subespecie y 1 variedad se localizan por primera vez en la provincia de Pontevedra (tabla 1). Asimismo, el catálogo incluye algunos taxa de interés corológico por su rareza o anómala distribución.

Respecto a la distribución en el continente europeo de los taxa localizados en la zona de estudio cabe destacar la presencia de Aspicilia calcarea, Buellia sardiniensis, Cladonia convoluta, Collema ryssoleum, Diploschistes diacapsis subsp. interpediens, Lecanora poeltiana, L. schistina, Parmelia tinctina, Petractis thelotremella y Protoparmelia montagnei, todos ellos, con un marcado carácter mediterráneo; su presencia no es sorprendente si consideramos la situación limítrofe de la 


\begin{tabular}{|c|c|c|c|c|c|c|c|c|c|c|}
\hline \multirow[t]{2}{*}{ Especies } & \multicolumn{10}{|c|}{ Localidades } \\
\hline & 1 & 2 & 3 & 4 & 5 & 6 & 7 & 8 & 9 & 10 \\
\hline Acrocordia macrospora Massal & & & & & + & & + & & & \\
\hline AAcrocordia salweyi (Nyl.) A.L. Sm. & & + & & & & & & & & \\
\hline Amandinea punctata (Hoffm.) Coppins \& Scheid. & & + & + & & & + & & + & & \\
\hline Anaptychia runcinata (With.) Laundon & & & + & + & + & + & + & + & & \\
\hline $\begin{array}{l}\text { Aspicilia caesiocinerea (Nyl. ex Malbr.) Arnold } \\
\text { var. caesiocinerea }\end{array}$ & & & & & & & & + & & \\
\hline Aspicilia caesiocinerea var. subdepressa (Nyl.) & & & & & & & & & & \\
\hline Clauz. \& Roux & & & + & & & + & & & + & \\
\hline AAspicilia calcarea (L.) Mudd & & + & & + & & & & & & \\
\hline$\checkmark$ Aspicilia contorta (Hoffm.) Krempelh & & & + & & & & & & & \\
\hline$\Delta$ Bacidia trachona (Ach.) Lettau & & + & & & & & & & & \\
\hline Buellia aethalea (Ach.) Th. Fr. & & & + & & + & & + & & + & \\
\hline$\downarrow$ Buellia ocellata (Flotow) Körber & & & + & + & & & & & & \\
\hline Buellia sardiniensis Steiner & & + & + & + & & + & + & & + & \\
\hline Buellia stellulata (Taylor) Mudd & & + & + & + & + & + & + & + & + & + \\
\hline Buellia subdisciformis (Leighton) Vainio & & & & & + & & & & & \\
\hline Caloplaca aractina (Fr.) Häyrén & & + & & & & & & & & \\
\hline Caloplaca aurantia (Pers.) Steiner & & + & & & & & & + & & \\
\hline *Caloplaca ceracea Laundon & & & & & & & & + & & \\
\hline Caloplaca citrina (Hoffm.) Th. Fr. & & + & & & & & & & & \\
\hline Caloplaca crenularia (With.) Laundon & & + & + & + & + & & + & + & & \\
\hline -Caloplaca crenulatella (Nyl.) H. Olivier & & & & & + & + & & & & \\
\hline Caloplaca littorea Tavares & & + & & & & + & + & + & & \\
\hline Caloplaca marina (Wedd.) Du Rietz & & + & & & + & + & + & + & & \\
\hline $\begin{array}{l}\text { Caloplaca aff. microthallina (Wedd.) Zahlbr. } \\
\text { Caloplaca thallincola (Wedd.) Du Rietz }\end{array}$ & & + & & & + & & + & + & & \\
\hline Caloplaca vitellinula auct. medieur. non H.Olivier & & & & & & + & & & & \\
\hline Candelariella vitellina (Hoffm.) Müll. Arg. & & + & + & + & + & + & + & + & + & \\
\hline -Catillaria atomarioides (Müll. Arg.) Kilias & & + & & & + & & + & & & \\
\hline Catillaria chalybeia (Borrer) Massal. & & + & + & + & + & + & + & + & & \\
\hline Catillaria chalybeia var. chloropolyza (Nyl.) Kilias & & + & & & + & & & & & \\
\hline -Catillaria lenticularis (Ach.)Th. Fr. & & & & & & + & & + & & \\
\hline Cetraria aculeata (Schreber) Fr. & & & & & & & & & + & \\
\hline Cladonia caespiticia (Pers.) Flörke & & & & + & & & & & & \\
\hline Cladonia convoluta (Lam.) Anders & + & & & & & & & & & \\
\hline Cladonia foliacea (Hudson) Willd. & & & & & & & & & + & \\
\hline Cladonia furcata (Hudson) Schrader & & & + & & + & + & & + & + & \\
\hline - Cladonia mediterranea Duvign. \& des Abb. & & & & & + & + & & & + & \\
\hline - Cladonia metacorallifera Asah. & & & & & & & & & + & + \\
\hline ACladonia pocillum (Ach.) O. J. Rich. & & & & & & + & & & & \\
\hline Cladonia pyxidata (L.) Hoffm. & & & + & & & & & + & & \\
\hline Cladonia rangiformis Hoffm. var. rangiformis & + & & + & & & & & & & \\
\hline Cladonia rangiformis var. pungens (Ach.) Vainio & + & & & + & & & & & & \\
\hline ACladonia rei Schaerer & & & + & & & & & & & \\
\hline Cladonia squamosa Scop. (Hoffm.) var. squamosa & & & & & & & & & & + \\
\hline -Cladonia squamosa var. subsquamosa & & & & & & & & & & \\
\hline (Leighton) Vainio & & & + & & & & & & & + \\
\hline ACladonia subrangiformis Sandst. & + & & & & & & & & & \\
\hline ACladonia symphycarpa (Flörke) Fr. & & & + & & & & & & + & + \\
\hline
\end{tabular}

Tabla 1. Distribución de las especies en las localidades muestreadas. Se destacan con símbolos las nuevas citas para España $(\bullet)$, para Galicia $(\mathbf{\Delta})$ y para la provincia de Pontevedra $(\diamond)$. 
Localidades

1

$\checkmark$ Collema auriforme (With.) Coppins \& Laundon +

-Collema cristatum (L.) Wigg.

Collema furfuraceum (Arnold) Du Rietz

$\checkmark$ Collema ryssoleum (Tuck.) A. Schneider

- Collema undulatum Flotow

Degelia atlantica (Degel.) P. M. Jørg. \& P. James

Dermatocarpon miniatum (L.) Mann

Diploicia canescens (Dickson) Massal.

Diploicia subcanescens (Werner) Haf. \& Poelt

$\checkmark$ Diploschistes actinostomus (Ach.) Zahlbr.

$\Delta$ Diploschistes diacapsis (Ach.) Lumbsch subsp. interpediens (Nyl.) Clauz. \& Roux

$\checkmark$ Diplotomma ambiguum (Ach.) Flagey

-Diplotomma chlorophaeum (Leight.) Szat

Fuscidea cyathoides (Ach.) V. Wirth \& Vèzda

Fuscidea lygaea (Ach.) V. Wirth \& Vèzda

Gyalideopsis $s p$.

Hypogymnia physodes (L.) Nyl.

Lasallia pustulata (L.) Mérat

Lecania aipospila (Wahlenb.) Th. Fr.

Lecania atrynoides Knowles

Lecania hutchinsiae (Nyl.) A. L. Sm.

$\Delta$ Lecania nylanderiana Massal.

Lecanora actophila Wedd.

Lecanora campestris (Schaerer) Hue

Lecanora dispersa (L.) Sommerf.

Lecanora gangaleoides Nyl.

Lecanora helicopis (Wahlenb.) Ach.

-Lecanora lecideoides (Nyl.) Harm.

Lecanora muralis (Schreber) Rabenh.

ALecanora poeltiana Clauz. \& Roux

- Lecanora schistina (Nyl.) Arnold

- Lecanora subcarnea (Liljeblad) Ach.

Lecanora sulphurea (Hoffm.) Ach.

Lecidea fuscoatra (L.) Ach.

Lecidea sarcogynoides Körber

Lecidella asema (Nyl.) Knoph \& Hertel

Lecidella scabra (Taylor) Hertel \& Leuckert

Lecidella viridans (Flotow) Körber

Lepraria incana (L.) Ach.

Leprocaulon microscopicum (Will.)

Gams ex D. Hawksw.

Leproloma cacuminum (Massal.) Laundon

Leptogium gelatinosum (With.) Laundon

Leptogium lichenoides (L.) Zahlbr.

var. lichenoides

Leptogium lichenoides

var. pulvinatum (Hoffm.) Zahlbr.

ALeptogium massiliense Nyl.

Leptogium microphylloides Nyl.

-Leptogium tenuissimun (Dickson) Körber

1

2

$+$

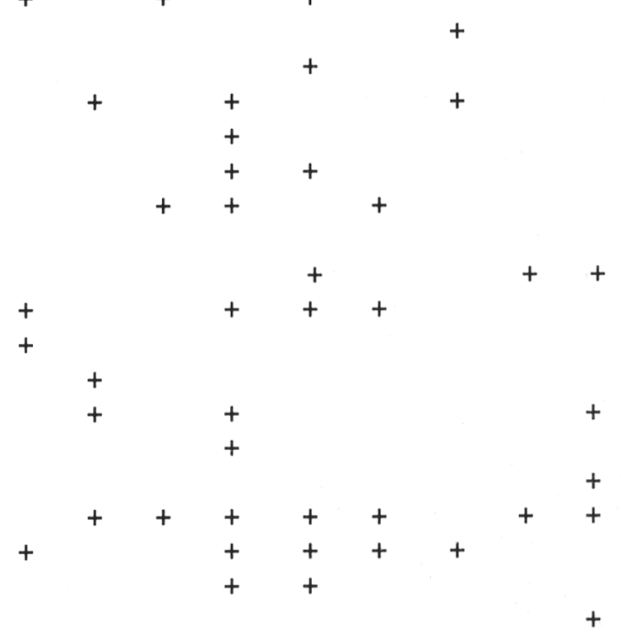

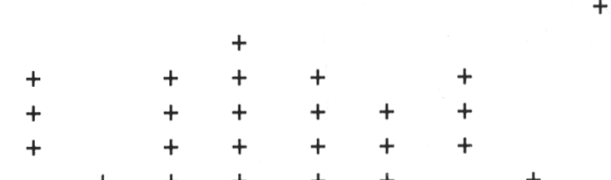

$+$
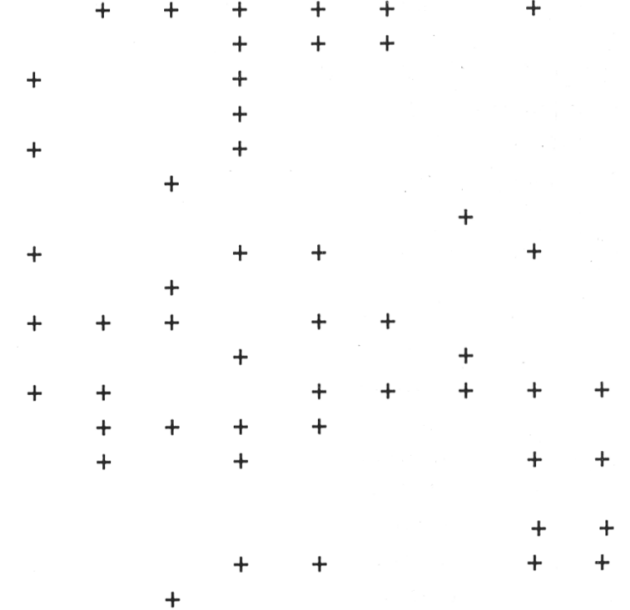

Tabla 1 (continuación). 


\begin{tabular}{clllllllllll}
\hline Especies & Localidades \\
\hline & 1 & 2 & 3 & 4 & 5 & 6 & 7 & 8 & 9 & 10
\end{tabular}

Leptogium teretiusculum (Wallr.) Arnold

Lichina confinis (O. F. Müller) Agardh

Lichina pygmaea (Lightf.) Agardh

- Nephroma tangeriense (Maheu \& Gillet) Zahlbr.

Normandina pulchella (Borrer) Nyl.

-Ochrolechia crozalsiana Clauz. \& Roux

Ochrolechia parella (L.) Massal.

Ochrolechia tartarea (L.) Massal.

Opegrapha caesarensis Nyl.

Opegrapha calcarea $\mathrm{Sm}$.

Opegrapha conferta Anzi

Parmelia caperata (L.) Ach.

Parmelia conspersa (Ach.) Ach.

Parmelia glabratula (Lamy) Nyl.

subsp. fuliginosa (Duby) Laundon

Parmelia horrescens Taylor

Parmelia pulla Ach.

Parmelia revoluta Flörke

Parmelia revoluta var. britannica

(Hawksw. \& P. James) V. Wirth

Parmelia subrudecta Nyl.

Parmelia sulcata Taylor

Parmelia tiliacea (Hoffm.) Ach.

Parmelia tinctina Maheu \& Gillet

Parmelia verruculifera Nyl.

-Parmeliopsis ambigua (Wulfen) Nyl.

Parmotrema chinense (Osbeck) Hale \& Ahti

Parmotrema reticulatum (Taylor) M. Choisy

Parmotrema stuppeum (Taylor) Hale

Pertusaria amara (Ach.) Nyl.

Pertusaria excludens Nyl.

APertusaria flavicans Lamy

Pertusaria gallica B. de Lesd.

Pertusaria lactescens Mudd

Pertusaria melanochlora (DC.) Nyl.

Pertusaria monogona Nyl.

Pertusaria pseudocoralina (Liljeblad) Arnold

- Pertusaria rupestris (DC.) Schaerer

APetractis thelotremella (Bagl.) Vèzda

Phaeophyscia orbicularis (Necker) Moberg

Physcia adscendens (Fr.) H. Olivier

Physcia caesia (Hoffm.) Fürnrohr

Physcia dubia (Hoffm.) Lettau

Physcia stellaris (L.) Nyl.

Physcia tenella (Scop.) DC.

Physcia tribacia (Ach.) Nyl.

Physcia tribacoides Nyl.

Polysporina simplex (Davies) Vèzda

Porina chlorotica (Ach.) Müll. Arg.

Porpidia albocaerulescens (Jacq.) Hertel \& Knoph

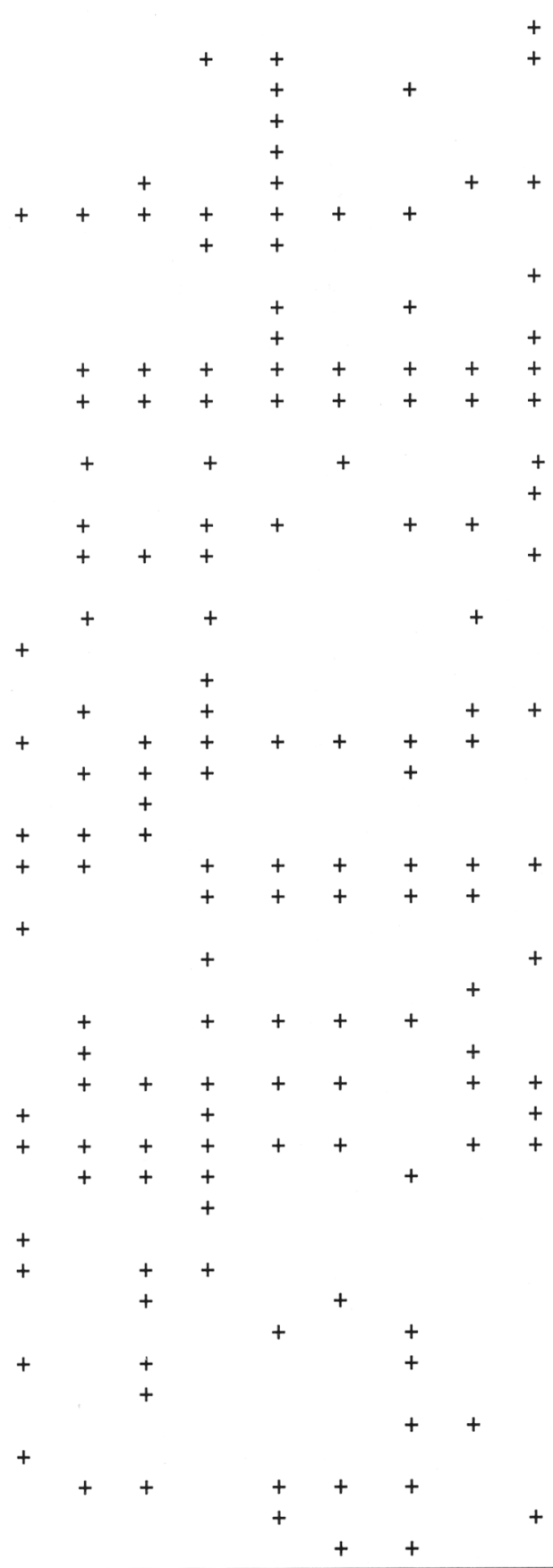

Tabla 1 (continuación). 
Especies

Porpidia cinereoatra (Ach.) Hertel \& Knoph Porpidia crustulata (Ach.) Hertel \& Knoph Porpidia macrocarpa (DC.) Hertel \& Schwab Protoparmelia montagnei (Fr.) Poelt \& Nimis Pyrenocollema halodytes (Nyl.) R. C. Harris Ramalina cuspidata (Ach.) Nyl.

Ramalina fenestrata Motyka

Ramalina siliquosa (Hudson) A. L. Sm. Ramalina subfarinacea (Crombie) Nyl.

$\Delta$ Rhizocarpon drepanodes Feuerer

Rhizocarpon lecanorinum Anders.

Rhizocarpon obscuratum (Ach.) Massal.

Rhizocarpon richardii (Nyl.) Zahlbr.

$\checkmark$ Rhizocarpon riparium Räsänen subsp. lindsayanum (Räs) Thoms.

Rinodina atrocinerea (Hooker) Körber

Rinodina beccariana Bagl.var. beccariana

Rinodina confragosa (Ach.) Körber

-Rinodina occulta (Körber) Sheard

Rinodina oxydata (Massal.) Massal.

ARoccella boergesenii Vainio

Roccella fuciformis (L.) DC.

Roccella phycopsis (Ach.) Ach.

$\checkmark$ Roccella tuberculata Vainio

Roccella vicentina (Vainio) Vainio

Sarcogine clavus (DC.) Krempelh.

-Sarcogine privigna (Ach.) Massal.

Scoliciosporum umbrinum (Ach.) Arnold

var. umbrinum

Scoliciosporum umbrinum

var. compactum (Körber) Clauz. \& Roux

- Sphinctrina leucopoda Nyl.

A Spilonema paradoxum Bornet

Thephromela atra (Hudson) Hafellner

-Thelidium minutulum Körber

*Thelotrema wightii (Taylor) Nyl.

$\Delta$ Thermutis vetulina (Ach.) Flotow

Toninia mesoidea (Nyl.) Zahlbr.

AToninia sedifolia (Scop.) Timdal

$\Delta$ Toninia squalida (Ach.) Massal.

Trapelia cf. coarctata (Sm.) M. Choisy

- Trapelia involuta (Taylor) Hertel

Cf. Trapeliopsis pseudogranulosa

Coppins \& P. James

Usnea cornuta Körber

Usnea rubicunda Stirton

Verrucaria amphibia Clemente

Verrucaria maura Wahlenb.

Verrucaria nigrescens Pers.

Verrucaria prominula $\mathrm{Nyl}$.

Xanthoria parietina (L.) Th. Fr.
Localidades
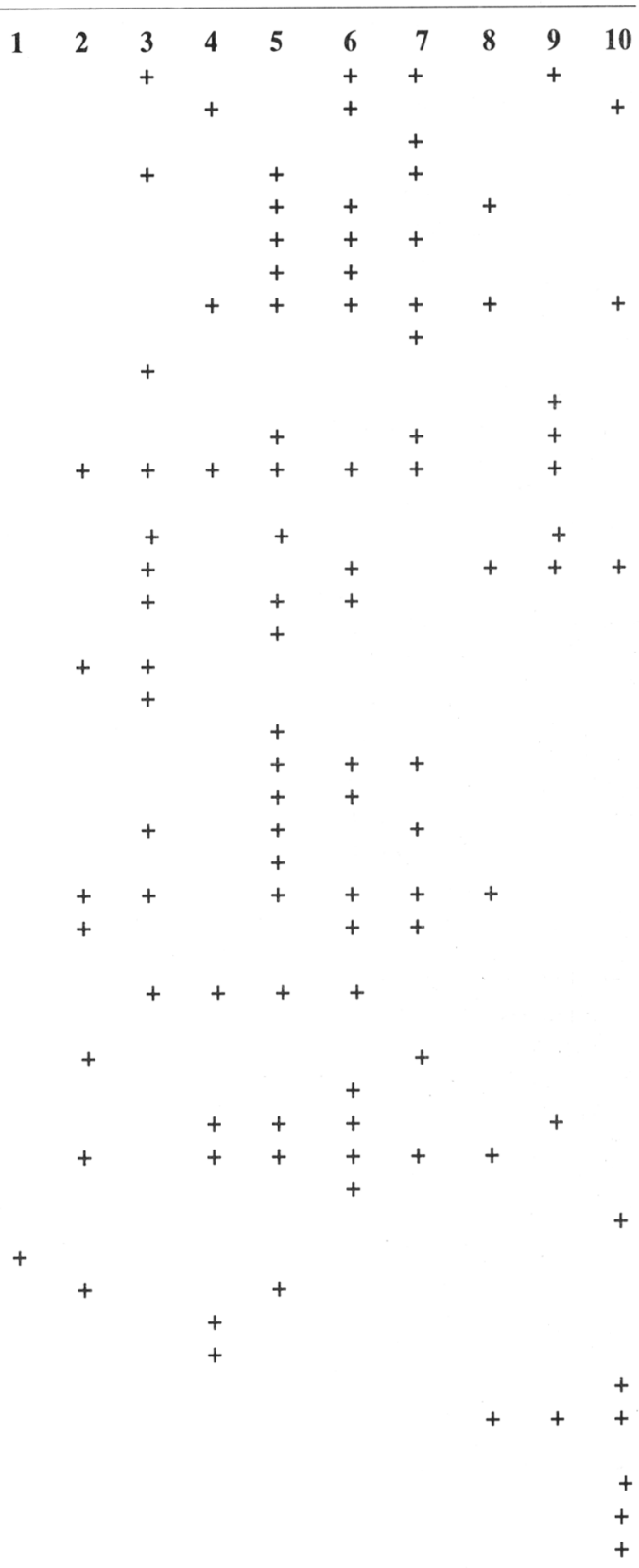

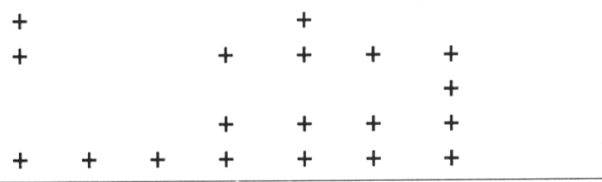

Tabla 1 (continuación). 
zona de estudio con esta región fitogeográfica.

La homogeneidad del substrato rocoso en la mayor parte del territorio no permite reconocer diferencias en cuanto al asentamiento de la vegetación liquénica; solamente, en algunos puntos del litoral, coexisten afloramientos de granodiorita en el supra- y adlitoral y gneises combinados con esquistos en el mesolitoral. Aún así se puede concluir que no parece existir especificidad por el tipo de roca y que el establecimiento de las especies parece estar condicionado por la influencia marina, teniendo en cuenta que han sido identificados una serie de taxa propios de substratos calcáreos (Acrocordia salweyi, Aspicilia calcarea, A. contorta, Catillaria lenticularis, Collema cristatum, C. ryssoleum, Leptogium tenuissimum, Petractis thelotremella y Toninia mesoidea) sobre rocas ácidas, cuya presencia se justifica por el enriquecimiento en sales que presentan las rocas del litoral. Algunas especies calcícolas especialmente exigentes fueron recolectadas exclusivamente sobre mortero.

El estudio de la zonación litoral ha permitido apreciar el predominio de Verrucaria maura, Caloplaca marina, C. thallincola y Lecania aipospila en la franja sometida a la acción de las mareas. En localidades expuestas se presentan, además, Lichina confinis y $L$. pygmaea, próximas al límite inferior de la marea.

La transición entre el supralitoral y el adlitoral se produce de forma gradual, y muchas de las especies identificadas son comunes a ambos niveles, si bien varía la cobertura de los talos. Se aprecia un predominio de las especies foliáceas y fruticulosas en el adlitoral, sobre los líquenes crustáceos que desarrollan talos mayores en el supralitoral. También es importante destacar que los líquenes detectados en ambos niveles mayoritariamente son especies nitrófilas, cuyo desarrollo se ve favorecido por la acción antropógena y las aves marinas, entre otros factores.

\section{BIBLIOGRAFÍA}

ÁLVAREZ, J.-1993- Flora y vegetación epifítica de la Sierrade O Caurel (Lugo). Tesis Doctoral. Universidad de Santiago de Compostela. Inédita.

ÁLVAREZ, J. y R. CARBALLAL - 1987- Fragmenta chorologica occidentalia, Lichenes, 1297-1351. Anales Jard. Bot. Madrid 45(1): 290-294.

ÁLVAREZ, J. y R. CARBALLAL - 1988- Fragmenta chorologica occidentalia, Lichenes, 896-916. Anales Jard. Bot. Madrid 44(1): 151-153.

AMO Y MORA, M. Del -1870- Flora criptogámica de la Península Ibérica (Líquenes):298-450. Granada.

BAHILLO, L., LÓPEZ DE SILANES, M.E. y CARBALLAL, R.-1987- Flora liquénica de los roquedos marítimos gallegos. Act. VI Simp. Nac. Bot. Cript.: 361-370.

BOQUERAS, M.-1993- Flora y vegetació dels líquens epifitics de les terres meridionals de Catalunya. Tesis Doctoral. Universidad de Barcelona. Inédita.

BOOM VAN DEN, P. P. G. \& A. GÓMEZ-BOLEA -1991 - Contribution to the lichen flora of Spain. Nova Hedwigia 53 (3-4): 497-505.

BURGAZ, A. R. \& T. AHTI -1994- Contribution to the study of genera Cladina and Cladonia in Spain II. Nova Hedwigia 59: 399-440.

CARBALLAL, R., I. BRAVO y M. E. LÓPEZ DE SILANES -1983- Novedades para la flora liquénica gallega. Lazaroa 5: 277-281.

CARBALLAL, R. y A. GARCÍA-MOLARES -1986Líquenes epifíticos como indicadores de la contaminación atmosférica I. Utilización del índice de pureza atmosférica (IPA) en la ciudad de Vigo (España). Revista de Biología 13: 33 41.

CARBALLAL, R. y A. GARCÍA-MOLARES -1987Fragmenta chorologica occidentalia, Lichenes, 917-927. Anales Jard. Bot. Madrid 44(1):154155.

CARBALLAL, R. y A. GARCÍA-MOLARES -198788- Líquenes epifíticos como indicadores de la contaminación atmosférica II. Utilización de una escala cualitativa en la ciudad de Vigo (España). Lazaroa 10: 243-251.

CARBALLAL, R. y A. GARCÍA-MOLARES -1988Acercamiento a las comunidades liquénicas epifíticas del entorno urbano de la ciudad de Pontevedra (NO de España). Bol. Soc. Brot., 
Sér.2, 61: 49-60.

CARBALLAL, R. y M. E. LÓPEZ DE SILANES 1985- Estudio base para la valoración de la contaminación atmosférica en la ciudad de Santiago mediante bioindicadores liquénicos. Trabajos Compostelanos de Biología 11:147154.

CARBALLAL, R., M. E. LÓPEZ DE SILANES y L. BAHILLO-1985- Fragmenta chorologica occidentalia, Lichenes, 46-72. Anales Jard. Bot. Madrid 42 (1): 236-238.

CLAUZADE, G. \& C. ROUX-1985- Likenoj de Okcidenta Europo. Ilustrita determinlibro. Bull. Soc. Bot. Centre-Ouest 7: 1-893.

CLAUZADE, G. \& C. ROUX -1987- Likenoj de Okcidenta Europo. Suplemento $2^{\mathrm{a}}$. Bull. Soc. Bot. Centre-Ouest 18: 177-214.

ClAuZADE, G. \& C. ROUX -1989- Likenoj de Okcidenta Europo. Suplemento $3^{\text {a }}$. Bull. Soc. Linn. Provence 40: 73-110.

COLMEIRO, M.-1889-Enumeración y revisión de las plantas de la Península Ibérica e Islas Baleares (Tomo V. Líquenes): 758-875.

ETAYO, J., M. E. LÓPEZ DE SILANES y L. BAHILLO -1991- Contribución a la flora liquénica de Galicia central - Tras os montes I. N.A.C.C.(Bioloxía) 2:31-39.

FISCHER-PIETTE, E. y J. SEOANE-CAMBA 1962- Examen écologique de la Ría de Camariñas. Bull. Inst. Océanogr. Monaco 61(1277): 1-38.

FOLLMAN, G.-1973- Beobachtungen zum Vorkommen spanischer Flechten I. Der formenkreis um Ramalina siliquosa (Huds.) A. L. Smith. Philippia 2(1): 3-12.

GIRALT, M. \& M. BARBERO -1995- The saxicolous species of the genus Rinodina in the Iberian Peninsula containing atranorin, pannarin or gyrophoric acid. Mycotaxon LVI: 45-80.

GIRALT, M., P.P.G. BOOM \& M. MATZER - 1997 The lichen genus Rinodina in Belgium, Luxembourg and Netherlands. Mycotaxon LXI: 103-151.

LANGE, J.-1860- Pugillus plantarum imprimis hispanicarum, quas in itinere 1851-52 legit Joh. Lange. Danks. Naturlist. Foren. Kjöbenhaure 1-4: 1-82.

LAUNDON, J.R.-1992-New British Species of Caloplaca. Lichenologist 24(1): 1-5.

LÓPEZ DE SILANES, M. E.-1992- Líquenes silicícolas de zonas húmedas, Pontedeume (La Coruña). Lazaroa 13:183-185.

LLIMONA, X.-1991- Història Natural dels Països Catalans. Fongs $i$ líquens. T.5. Fundació Enciclopèdia Catalana. Barcelona.

MAYRHOFER, H., M. MATZER, J. SATTLER \& J.M. EGEA - 1993- A revision of the AtlanticMediterranean Rinodina beccariana and related taxa (lichenized Ascomycetes, Physciaceae). Nova Hedwigia 57 (3-4): 281-304.

NAVARRO-ROSINÉS, P.-1992-Els liquens $i$ els fongs liquenicoles dels substrats carbonatats de Catalunya meridional. Tesis Doctoral. Universidad de Barcelona. Inédita.

NIMIS, P.L.-1993- The lichens of Italy. An annotated catalogue. Museo Regionale di Scienze Naturali. Torino.

NYLANDER, W.-1888- Lichens du nord du Portugal. Bol. Soc. Brot. IV: 1-53.

PAZ-BERMÚDEZ, G.-1998- Líquenes saxícolas e fungos liquenicolas da costa de Galicia. Tesis Doctoral. Universidad de Santiago de Compostela. Inédita.

PÉREZ, C., M. E. LÓPEZ DE SILANES y R. CARBALLAL -1991- Fragmenta chorologica occidentalia, Lichenes, 3433-3466. Anales Jard. Bot. Madrid 49(1):110-113.

PÉREZ-CIRERA, J.L.-1976- Tipos de vegetación bentónica y cormofítica litoral del Noroeste de España (Ría de Corme y Lage). Documents Phytosociologiques 15-18: 87-122.

PRIETO, B., T. RIVAS, B. SILVA, R. CARBALLAL \& M. E. LÓPEZ DE SILANES -1994Colonitation by lichens of granite dolmens in Galicia (NW Spain). International Biodeterioration \& Biodegradation: 47-60.

PRIETO, B., T. RIVAS, B. SILVA, R. CARBALLAL \& M. J. SÁNCHEZ -BIEZMA -1995- Étude écologique de la colonitation lichénique des églises des environs de Saint-Jaques-deCompostelle (NW Espagne). Criptogamie, Bryol. Lichénol. 16(3): 219-228.

POELT, J.-1988- Rhizocarpon Ram. em. Th. Fr. Subgen. Rhizocarpon in Europe. Artic and Alpine Research 20(3): 292-298.

PURVIS, O.W., B.J. COPPINS, D.L HAWKSWORTH, P.M. JAMES \& D.M. MOORE -1992- The Lichen Flora of Great Britain and Ireland. Natural History Museum Publications in association with The British 
Lichen Society. London.

PURVIS, O.W., P.M. JØRGENSEN \& P.M. JAMES -1995- The lichen genus Thelotrema Ach. in Europe. In: Farkas, E.E., Lucking, R. \& V. Wirth (eds.): Scripta LichenologicaLichenological papers dedicated to Antonín Vèzda. Bibl. Lichenol., 58: 335-360. J. Cramer, Berlín-Stuttgart.

SAMPAIO, G. y L. CRESPÍ -1927- Líquenes de la provincia de Pontevedra. Bol. R. Soc. Esp. Hist. Nat. 24: 135-151.

SÁNCHEZ-BIEZMA, M.J.-1997-Liquenes saxícolas de la sierra de A Capelada (A Coruña). Tesis Doctoral. Universidad de Santiago de Compostela. Inédita.

SEOANE-CAMBA, J.-1969-Sobre la zonación del sistema litoral y su nomenclatura. Inv. Pesqu. 33(1):261-267.

TAVARES, C.N.-1956- Lichenes seleccti exsiccati. Inst. Bot. Univ. Olisiponensis, Fasc. IV: 1-4.

TIMDAL, E.-1991- A monograph of the genus Toninia (Lecideaceae, Ascomycetes). Opera Botanica 110: 1-137.

VALCÁRCEL, C. P.-1994- Flora liquénica del municipio de A Fonsagrada (Lugo). Tesis Doctoral. Universidad de Santiago de Compostela. Inédita.
VALCÁRCEL, C. P., M. E. LÓPEZ DE SILANES y R. CARBALLAL - 1991-Los géneros Cladina y Cladonia (Lecanorales) en el municipio de Fonsagrada (Lugo). N.A.C.C. (Bioloxía) 2: 1321.

VALCÁRCEL, C. P., M. E. LÓPEZ DE SILANES y R. CARBALLAL - 1992- Fragmenta chorologica occidentalia, Lichenes, 4294-4335. Anales Jard. Bot. Madrid 50(2): 251-253.

Aceptado para su publicación en Octubre de 1999

Dirección de los autores. Departamento Biología Vegetal y Ciencia del Suelo. Facultad de Ciencias. Universidad de Vigo. Campus Lagoas Marcosende. 36200-VIGO (Pontevedra) 